\title{
LETTER
}

Acute myeloid leukemia

\section{Functional and clinical characterization of the alternatively spliced isoform AML1-ETO9a in adult patients with translocation $\mathrm{t}(8 ; 21)(\mathrm{q} 22$; q22.1) acute myeloid leukemia (AML)}

\author{
Mridul Agrawal ${ }^{1}$ - Peggy Schwarz ${ }^{2}$ - Benedetto Daniele Giaimo ${ }^{3}$ Ivan Bedzhov ${ }^{4}$ Andrea Corbacioglu ${ }^{1}$. \\ Daniela Weber ${ }^{1}$ - Verena I. Gaidzik ${ }^{1}$ - Nikolaus Jahn ${ }^{1}$ • Frank G. Rücker ${ }^{1}$ - Thomas Schroeder ${ }^{5} \cdot$ Thomas Kindler $^{6}$. \\ Mohammed Wattad ${ }^{7} \cdot K_{\text {Katharina Götze }}^{8} \cdot$ Michael Lübbert $^{9} \cdot$ Hans Salwender $^{10} \cdot$ Mark Ringhoffer $^{11}$. \\ Elisabeth Lange ${ }^{12}$. Elisabeth Koller ${ }^{13}$. Felicitas Thol ${ }^{14} \cdot$ Michael Heuser $^{14}$ - Arnold Ganser ${ }^{14} \cdot$ Lars Bullinger $^{15}$. \\ Peter Paschka ${ }^{1} \cdot$ Hartmut Döhner $^{1} \cdot$ Hartmut Geiger $^{16,17} \cdot$ Tilman Borggrefe $^{3} \cdot$ Konstanze Döhner $^{1} \cdot$ Franz Oswald $^{2}$
}

Received: 15 May 2019 / Revised: 29 May 2019 / Accepted: 6 June 2019 / Published online: 28 August 2019

(c) The Author(s) 2019. This article is published with open access

\section{To the Editor:}

Acute myeloid leukemia (AML) encompassing translocation $\mathrm{t}(8 ; 21)(\mathrm{q} 22 ; \mathrm{q} 22.1)$ results in the chimeric fusion protein

These authors contributed equally: Mridul Agrawal, Peggy Schwarz

These authors jointly supervised this work: Konstanze Döhner, Franz Oswald

Supplementary information The online version of this article (https:// doi.org/10.1038/s41375-019-0551-4) contains supplementary material, which is available to authorized users.

Konstanze Döhner

konstanze.doehner@uniklinik-ulm.de

1 Klinik für Innere Medizin III, Universitätsklinikum Ulm, Ulm, Germany

2 Klinik für Innere Medizin I, Universitätsklinikum Ulm, Ulm, Germany

3 Institute of Biochemistry, University of Giessen, Giessen, Germany

4 Embryonic Self-Organization research group, Max Planck Institute for Molecular Biomedicine, Röntgenstraße 2048149 Münster, Germany

5 Klinik für Hämatologie, Onkologie und Klinische Immunologie, Universitätsklinikum Düsseldorf, Düsseldorf, Germany

6 III. Medizinische Klinik und Poliklinik, Universitätsmedizin Mainz, Mainz, Germany

7 Klinik für Hämatologie, Internistische Onkologie und Stammzellentransplantation, Evangelisches Krankenhaus EssenWerden, Essen, Germany

8 III. Medizinische Klinik, Klinikum rechts der Isar der Technischen Universität München, München, Germany
AML1-ETO (AE), also known as RUNX1-RUNX1T1 transcript. The presence of $\mathrm{AE}$ defines a precursor stage of leukemia, however additional molecular events are required for transformation [1,2]. Alternative splicing of the ETO gene introduces an additional exon adjacent to exon 8 , namely exon 9a, spanning $155 \mathrm{bp}$. Inclusion of exon 9a alters the open reading frame of the $\mathrm{AE}$ gene leading to a carboxy-terminal truncated isoform of the $\mathrm{AE}$ protein, known as AML1-ETO9a (AE9a), which lacks Drosophila nervy homology regions (NHR) 3 and 4 [3]. In a retroviral transduced mouse model, co-expression of AE and AE9a induces a more immature leukemic phenotype with a rapid

9 Klinik für Innere Medizin I, Universitätsklinikum Freiburg, Freiburg, Germany

10 II. Medizinische Abteilung, Asklepios Klinik Altona, Hamburg, Germany

11 Medizinische Klinik III, Städtisches Klinikum Karlsruhe, Karlsruhe, Germany

12 Klinik für Hämatologie, Onkologie und Palliativmedizin, Evangelisches Krankenhaus Hamm, Hamm, Germany

13 Medizinische Abteilung, Hanusch-Krankenhaus der WGKK, Wien, Austria

14 Klinik für Hämatologie, Hämostaseologie, Onkologie und Stammzelltransplantation, Medizinische Hochschule Hannover, Hannover, Germany

15 Medizinische Klinik m. S. Hämatologie, Onkologie und Tumorimmunologie, Campus Virchow-Klinikum, Charité Universitätsmedizin Berlin, Berlin, Germany

16 Institut für Molekulare Medizin, Universität Ulm, Ulm, Germany

17 Division of Experimental Hematology and Cancer Biology, CCHMC, Cincinnati, OH, USA 
onset of AML [4]. The authors hypothesized that the relative $A E 9 a$ allelic burden as compared to the full-length $A E$ transcript might affect the transforming capacity of the protein [4]. As to now, there is only scarce data on the incidence of AE9a with limited evidence indicating that $A E 9 a$ transcript levels (TL) impact on prognosis in $\mathrm{t}(8 ; 21)$ AML. In a previous study on 118 pediatric and adult $\mathrm{t}(8 ; 21)$-AML patients, elevated $A E 9 a(n=86)$ was correlated with the worsened clinical outcome as well as increased incidence of KIT mutations and higher KIT gene expression [5]. However, the two-step nested PCR approach used to detect $A E 9 a$ does not allow accurate quantification and therefore possibly overestimates gene expression levels. In another study, Ommen et al. reported the presence of $A E 9 a$ in $11 / 13$ patients with $\mathrm{t}(8 ; 21)$-AML and observed lower decline of $A E 9 a$ TL in relapsing as compared to nonrelapsing patients during the course of the disease [6]. More recently, we performed a transcriptome study applying novel high-throughput sequencing technologies and detected the $A E 9 a$ variant in 27/27 $\mathrm{t}(8 ; 21)$-AML cases [7].

$\mathrm{We}$, therefore, sought to systematically assess the incidence and prognostic significance of $A E 9 a$ co-expression in the context of clinical and genetic factors in a large clinically well-annotated cohort of patients with $\mathrm{t}(8 ; 21)$-AML. We complemented these analyses by the generation of a mouse model (Rosa26-LSL-AE9a-IRES-GFP x Vav1-Cre) with hematopoietic-specific AE9a-expression starting early on in development to determine the role of AE9a for leukemia initiation and progression.

In total, 129 patients based on the availability of a diagnostic bone marrow (BM) or peripheral blood (PB) sample were included; 93 patients were enrolled on one of five clinical trial protocols of the German-Austrian AML Study Group (AMLSG) (Supplemental Appendix); 36 patients were treated outside clinical studies. 127 patients received standard intensive chemotherapy and 2 patients were treated based on a non-intensive treatment protocol. The median follow up was 3.6 years (detailed patients characteristics are provided in the Supplemental Appendix, Table S1). AE9a mRNA expression was determined by qRT-PCR (Fig. S1a). Co-expression of $A E 9 a$ as a fraction of the full-length $A E$ transcript was reported as $A E 9 a / A E$ ratio (\%); $A B L 1$ was used as housekeeping gene control [8]. Gene mutation status was available for KIT, FLT3 (ITD/ TKD), NRAS and ASXL2 [9-11]. This study was conducted in accordance with the Declaration of Helsinki. Written informed consent for treatment and genetic testing was obtained from all patients.

The AE9a isoform was detectable in all 129 patients of our study cohort, which is in line with our recent finding and the data published by Ommen et al. [6, 7]. In contrast, Jiao et $a l$. identified the $A E 9 a$ splice variant only in a proportion of the patients [5]. This discrepancy is probably due to the varying techniques that have been applied; Jiao et al. determined the relative gene expression $<10^{-3}$ on a gradient dilution of Kasumi-1 cells as threshold for PCRnegativity [5], whereas our definition of PCR-negativity was set $\mathrm{C}_{\mathrm{t}}>\mathrm{Y}$-intercept. In our data set, the median $A E 9 a$ / $A E$ ratio was $32 \%$ (range $3-77 \%$ ) and did not significantly differ between $\mathrm{BM}(n=116$, range $8-77 \%$, median $31 \%)$ and $\mathrm{PB}(n=13$, range 3-66\%, median 52\%). Interestingly, the allelic $A E 9 a$ burden corresponded to our previous findings independently obtained by RNA-sequencing (13-64\%) [7]. Median AE9alAE ratio was neither correlated with clinical features (sex, age, WBC, platelets, BM blasts; Table S2) nor gene mutations affecting KIT, FLT3 or ASXL2.

Using Cox regression analysis, $A E 9 a / A E$ ratios were not associated with the clinical endpoints overall survival (OS), event-free survival (EFS) and cumulative incidence of relapse (CIR) (Table S3). The same was true when we performed univariate analyses comparing $A E 9 a / A E$ ratios dichotomized at the median ( $A E 9 a / A E^{>\text {median }}$ vs. $A E 9 a /$ $\left.A E^{\text {smedian }}\right)$ : here, $A E 9 a / A E$ ratios did not impact 4-yr OS (71 vs $79 \% ; P=0.37$; Fig. 1 left, Table S4), 4-yr EFS (67 vs $59 \% ; P=0.83$; Fig. 1 right, Table S4), and 4 -yr CIR (32 vs 33\%; $P=0.35$; Table S4). Furthermore, we evaluated a possible correlation between $A E 9 a / A E$ ratio and NRAS or KIT mutations that frequently co-occur in the $\mathrm{t}(8 ; 21)$-AML subtype. In a subgroup analysis, no significant differences with regard to the clinical endpoints OS, EFS and CIR were found between $A E 9 a / A E^{>\text {median }}$ and $A E 9 a / A E^{\leq \text {median }}$ if stratified according to NRAS (Fig. S1b) or KIT (Fig. S1c) mutations. Finally, absolute $A E 9 a$ quantification as ratio to the $A B L$ housekeeping gene was performed to test its prognostic value independently of the $A E$ transcript. $A E 9 a /$ $A B L$ ratios were dichotomized along the median ( $A E 9 a$ al $A B L^{>\text {median }}$ vs. $\left.A E 9 a / A B L^{\text {smedian }}\right)$ but again were not associated with outcome: 4 -yr OS (74 vs $89 \% ; P=0.33$ ), 4-yr EFS (58 vs $72 \% ; P=0.18$ ), and 4 -yr CIR (40 vs $29 \% ; P=$ $0.31)$.

Recently, several studies showed that elevated expression of AE9a in primitive hematopoietic cells in mice via retroviral transduction protocols and subsequent transplantation of the transduced cells results in leukemia, even though with a long latency $[12,13]$. These data imply a role for AE9a in leukemia initiation or progression, but do not exclude that additional mutations upon virus insertion might be necessary to contribute to the disease. In order to validate our clinical observations, we generated a novel mouse model for targeted expression of AE9a in the hematopoietic system (Rosa26-LSL-AE9a-IRES-GFP x Vav1-Cre, hereafter referred to as $A E 9 a$ mice, Fig. S2a-c, Tables S5-S7) to further characterize the role of AE9a in leukemogenesis independent of viral transduction protocols. Expression of $A E 9 a$ was verified at the level of mRNA (Fig. S2d), protein 

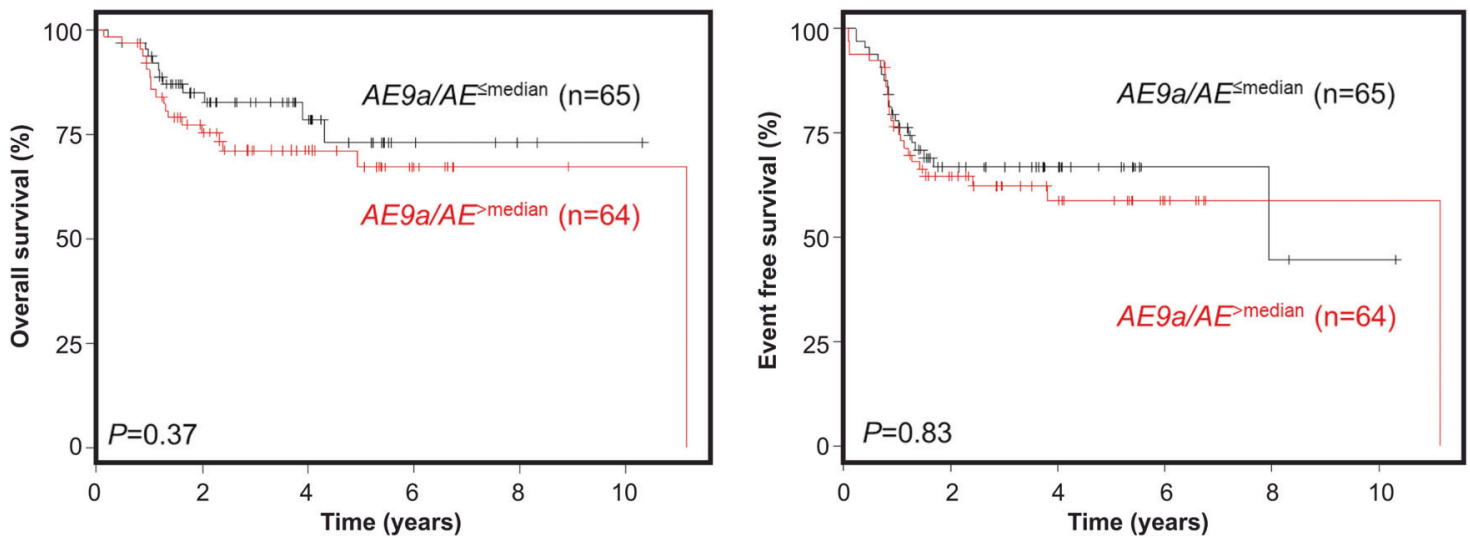

Fig. 1 Prognostic impact of $A E 9 a / A E$ on clinical outcome. OS (left) and EFS (right) are shown according to dichotomization of $A E 9 a / A E^{>m e d i a n}$ (red) and $A E 9 a / A E^{\leq \text {median }}$ (black)

a

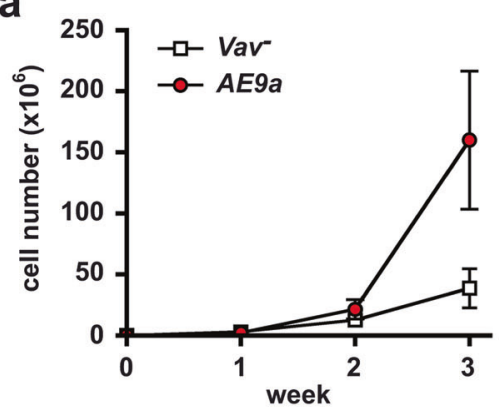

b

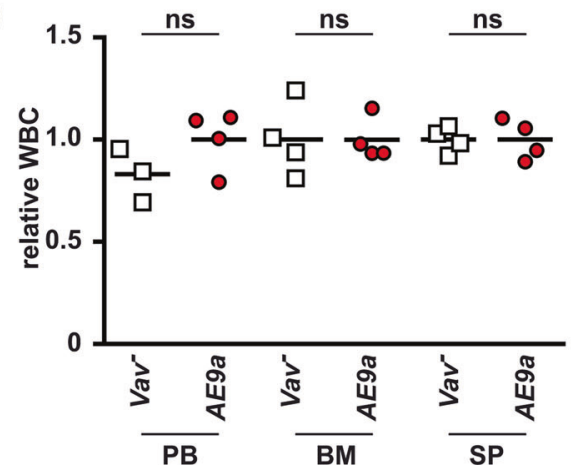

Fig. $2 A E 9 a$-expressing bone marrow cells exhibit enhanced stem cell characteristics but do not initiate leukemogenesis. a, left, $A E 9 a-$ expressing cells show enhanced proliferation capacity. Proliferation potential of Lineage ${ }^{-}, \mathrm{cKit}^{+}, \mathrm{GFP}^{+}$cells from 12 weeks old $A E 9 a$ mice (red dots) and Lineage ${ }^{-}, \mathrm{cKit}^{+}$cells isolated from Cre-negative littermate controls $\left(\mathrm{Vav}^{-}\right.$, white squares) in suspension culture was estimated by cell number counts taken in seven days intervals over 3 weeks. $\mathrm{MW} \pm \mathrm{SD}, n=2$. a, right, $\mathrm{AE9}$ a-expressing cells show significant self-renewal capacity. Colony-forming potential of Lineage ${ }^{-}$, $\mathrm{cKit}^{+}, \mathrm{GFP}^{+}$cells from 12 weeks old $A E 9 a$ mice (red bars) and Lineage $^{-}, \mathrm{cKit}^{+}$cells isolated from $\mathrm{Vavl}^{-}$littermate controls (white bars) was measured by serial replating on semi-solid methylcellulose

(Fig. S2e) as well as by GFP co-expression (Fig. S2f, g). As expected, isolated $\mathrm{GFP}^{+} \mathrm{BM}$ cells from twelve weeks old AE9a mice (Fig. S3a) showed increased proliferation rates
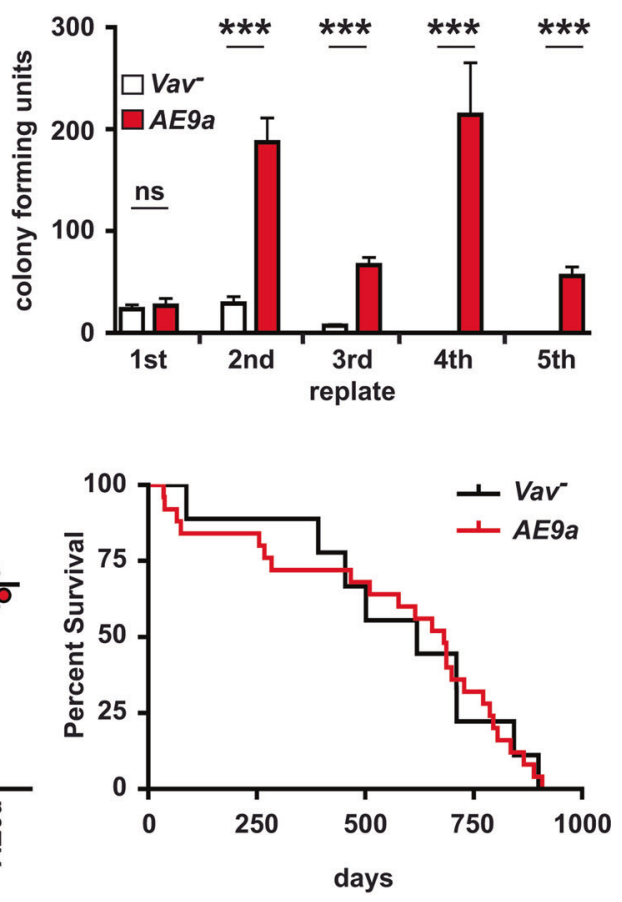

medium in seven days intervals over 5 weeks. $\mathrm{MW} \pm \mathrm{SD}$ (error bars) of the colony-forming units of triplicates from one representative experiment with $n=2$ mice/group is shown. $\mathbf{b}$, left, White blood cell counts (WBC) are not altered in 16 weeks old $A E 9 a$ mice (red dots) compared to $\mathrm{Vav}^{-}$littermate controls (white squares). Individual and mean values of peripheral blood (PB), bone marrow (BM) and spleen (SP) from $n=4$ mice/group are shown relative to the mean of the respective $\mathrm{Vav}^{-}$group. (b, right) $A E 9 a$ expression in the hematopoietic compartment does not influence survival of mice. Kaplan-Meier plot illustrating that survival in $A E 9 a$ mice (red line, mean survival 580 days, $n=25$ ) is not altered compared to $\mathrm{Vav}^{-}$control mice (black line, mean survival 559 days, $n=9$ ). ns, not significant; $* * * p<0.001$

(Fig. 2a, left) and colony-forming capacity (Fig. 2a, right) compared to cells from control $\left(\mathrm{Vav}^{-}\right)$mice. In addition, numbers of short term hematopoietic stem cells (ST-HSCs, 
Fig. S3b, left) were significantly elevated, while there were no significant changes in the number of long term hematopoietic stem cells (LT-HSCs, Fig. S3b, middle) and LSK cells (Fig. S3b, right). In addition, the frequency of CMPs (common myeloid progenitors; CD16/32 $2^{-}, \mathrm{CD}^{+} 4^{+}$) and GMPs (granulocyte/macrophage progenitors, CD16/32+, $\mathrm{CD}^{+} 4^{+}$), but not MEPs (megakaryocyte-erythrocyte progenitors, CD16/32- $2^{-}$CD34 ${ }^{-}$) was elevated (Fig. S3c). Most interestingly, although there was a tendency for an elevated number of $\mathrm{c}-\mathrm{Kit}^{+}$cells in BM and spleen which usually correlates with pre-leukemia, [Fig. S3d [12, 13]], none of the AE9a-expressing mice showed signs of leukemia as revealed by WBC (Fig. 2b, left) or survival compared to the controls (Fig. 2b, right).

We here report on the occurrence and prognostic impact of the $A E 9 a$ splice variant in the so far largest set of adult t $(8 ; 21)$-AML patients. Using a sensitive and robust quantitative RT-PCR assay, $A E 9 a$ was detectable in all patients. In contrast to the previous studies, our study was performed in a large cohort of uniformly treated patients. Neither $A E 9 a /$ $A E$, nor $A E 9 a / A B L$ ratios correlate with any clinical feature and they do not impact on clinical outcome. These clinical observations are in line with data generated by our novel murine model which unequivocally demonstrates that expression of AE9a might contribute to leukemogenesis but is clearly not sufficient for the initiation of leukemia in mice.

We have recently investigated the molecular mechanisms of AE9a-dependent transformation in a viral transduction/ transplantation model by analyzing its dual role in deregulation of the AML1 activating and the ETO repressing gene regulatory functions. In that system, the deregulation of both Notch and Amll target genes were required for the development of AE9a-driven leukemia [12] further supporting a necessary, but not sufficient role of AE9a for leukemia initiation. Thus, it is likely that a viral integration vector system for introducing AE9a in mice may cause leukemia through the activation of adjacent protooncogenes and therefore might not adequately recapitulate the human leukemogenesis.

In summary, in our large cohort of adult patients with $\mathrm{t}(8 ; 21)$-AML alternative splicing of the AML1-ETO fusion transcript represents a common feature. We could demonstrate that the allelic $A E 9 a$ burden does not impact prognosis of this AML subtype therefore precluding its potential as a novel independent prognostic marker. Our clinical observation data were complemented by our recently established conditional AE9a knock-in mouse model showing that AE9a expression leads to enhanced proliferation and replating capacity but not to overt leukemia. Thus, AE9a rather acts as a precondition which requires a "second hit" for the development of AML. Alternative model systems like our tissue-specific knock-in mouse model may help to identify the critical "second hit" or additional environmental factors such as irradiation or chemotherapeutic agents.

Acknowledgements We are grateful to Drs. H. Jumaa, M. Bach, E. Surova and M. Stemmler for sharing reagents and knowledge. We want to thank E. Lopez and Dr. B. Kanzler from the Transgenic Mouse Unit of the Max Planck Institute in Freiburg (Germany) for the generation of the knock-in mice. This work was in part supported by the collaborative research grant TRR81 and BO 1639/9-1 by the DFG (German Research Foundation), the Max Planck Society and the EXC 294 in Freiburg and the Excellence Cluster for Cardio Pulmonary System (ECCPS) in Giessen to TB. BDG is supported by a Research Grant of the University Medical Center Giessen and Marburg (UKGM). This study was supported in part by the Excellence Initiative of the German Federal and State Governments (GSC-4, Spemann Graduate School). This study was additionally supported in part by the Deutsche Forschungsgemeinschaft (SFB 1074 project; subproject A3 and B3). MA was supported by the Else Kröner-Forschungskolleg and the Deutsche Forschungsgemeinschaft (DFG, AG252/1-1). We greatly acknowledge the members of the German-Austrian AML Study Group (AMLSG) for providing patient samples and clinical information.

Author contribution MA, PS, TB, HG, LB, HD, KD, and FO designed the study and wrote the manuscript; BDG and IB generated the knock-in mouse model; MA, PS, AC, VIG, FGR, and NJ performed the experiments; MA, AC, DW, HD, PS, FO, and KD analyzed the results; DW performed statistical analyses; MA, AC, VIG, NJ, FGR, TS, TK, MW, KG, ML, HS, MR, EL, EK, FT, MH, AG, LB, PP, $\mathrm{HD}$, and KD provided patient samples and clinical information.

\section{Compliance with ethical standards}

Conflict of interest The authors declare that they have no conflict of interest.

Publisher's note: Springer Nature remains neutral with regard to jurisdictional claims in published maps and institutional affiliations.

Open Access This article is licensed under a Creative Commons Attribution 4.0 International License, which permits use, sharing, adaptation, distribution and reproduction in any medium or format, as long as you give appropriate credit to the original author(s) and the source, provide a link to the Creative Commons license, and indicate if changes were made. The images or other third party material in this article are included in the article's Creative Commons license, unless indicated otherwise in a credit line to the material. If material is not included in the article's Creative Commons license and your intended use is not permitted by statutory regulation or exceeds the permitted use, you will need to obtain permission directly from the copyright holder. To view a copy of this license, visit http://creativecommons. org/licenses/by/4.0/.

\section{References}

1. Higuchi M, O'Brien D, Kumaravelu P, Lenny N, Yeoh EJ, Downing JR. Expression of a conditional AML1-ETO oncogene bypasses embryonic lethality and establishes a murine model of human $\mathrm{t}(8 ; 21)$ acute myeloid leukemia. Cancer Cell. 2002;1:63-74.

2. Schessl C, Rawat VP, Cusan M, Deshpande A, Kohl TM, Rosten PM, et al. The AML1-ETO fusion gene and the FLT3 length 
mutation collaborate in inducing acute leukemia in mice. J Clin Invest. 2005;115:2159-68.

3. Wolford JK, Prochazka M. Structure and expression of the human MTG8/ETO gene. Gene. 1998;212:103-9.

4. Yan M, Kanbe E, Peterson LF, Boyapati A, Miao Y, Wang Y, et al. A previously unidentified alternatively spliced isoform of $t$ $(8 ; 21)$ transcript promotes leukemogenesis. Nat Med. 2006;12:945-9.

5. Jiao B, Wu CF, Liang Y, Chen HM, Xiong SM, Chen B, et al. AML1-ETO9a is correlated with C-KIT overexpression/mutations and indicates poor disease outcome in $\mathrm{t}(8 ; 21)$ acute myeloid leukemia-M2. Leukemia. 2009;23:1598-604.

6. Ommen HB, Ostergaard M, Yan M, Braendstrup K, Zhang DE, Hokland P. Persistent altered fusion transcript splicing identifies RUNX1-RUNX1T1 + AML patients likely to relapse. Eur J Haematol. 2010;84:128-32.

7. Faber ZJ, Chen X, Gedman AL, Boggs K, Cheng J, Ma J, et al. The genomic landscape of core-binding factor acute myeloid leukemias. Nat Genet. 2016;48:1551-6.

8. Krönke J, Schlenk RF, Jensen KO, Tschurtz F, Corbacioglu A, Gaidzik VI, et al. Monitoring of minimal residual disease in NPM1-mutated acute myeloid leukemia: a study from the
German-Austrian acute myeloid leukemia study group. J Clin Oncol. 2011;29:2709-16.

9. Jahn N, Agrawal M, Bullinger L, Weber D, Corbacioglu A, Gaidzik VI, et al. Incidence and prognostic impact of ASXL2 mutations in adult acute myeloid leukemia patients with $t(8 ; 21)$ (q22;q22): a study of the German-Austrian AML Study Group. Leukemia. 2017;31:1012-5.

10. Paschka P, Du J, Schlenk RF, Gaidzik VI, Bullinger L, Corbacioglu $\mathrm{A}$, et al. Secondary genetic lesions in acute myeloid leukemia with $\operatorname{inv}(16)$ or $t(16 ; 16)$ : a study of the German-Austrian AML Study Group (AMLSG). Blood. 2013;121:170-7.

11. Schlenk RF, Döhner K, Krauter J, Frohling S, Corbacioglu A, Bullinger L, et al. Mutations and treatment outcome in cytogenetically normal acute myeloid leukemia. $\mathrm{N}$ Engl $\mathrm{J}$ Med. 2008;358:1909-18.

12. Thiel VN, Giaimo BD, Schwarz P, Soller K, Vas V, Bartkuhn M, et al. Heterodimerization of AML1/ETO with CBFbeta is required for leukemogenesis but not for myeloproliferation. Leukemia. 2017;31:2491-502.

13. Yan M, Ahn EY, Hiebert SW, Zhang DE. RUNX1/AML1 DNAbinding domain and ETO/MTG8 NHR2-dimerization domain are critical to AML1-ETO9a leukemogenesis. Blood. 2009;113:883-6. 